\title{
Impact of operator interference in dark matter direct detection experiments
}

\author{
A. Brenner, ${ }^{a, b, *}$ G. Herrera, ${ }^{a, b}$ A. Ibarra, ${ }^{b}$ S. Kang, ${ }^{c}$ A. Rappelt, ${ }^{b}$ S. Scopel ${ }^{c}$ and G. \\ Tomar $^{b}$ \\ ${ }^{a}$ Max-Planck-Institut für Physik (Werner-Heisenberg-Institut), \\ Föhringer Ring 6, 80805 München, Germany \\ ${ }^{b}$ Physik-Department, Technische Universität München, \\ James-Franck-Straße, 85748 Garching, Germany \\ ${ }^{c}$ Department of Physics, Sogang University, \\ Seoul 121-742, South Korea \\ E-mail: anja.brenner@tum.de, gonzalo.herrera@tum.de, ibarra@tum.de, \\ francis735@naver.com, andreas.rappelt@tum.de, scopel@sogang.ac.kr, \\ physics.tomar@tum.de
}

For dark matter particles with spin up to $1 / 2$, the non-relativistic effective field theory provides 28 coupling strengths for the dark matter-nucleon interactions. The standard assumption of collaborations is that dark matter couples equally to protons and neutrons. Since this is not necessarily true, we developed a method to derive limits taking the interference among operators into account. Using this method and data provided from XENON1T and PICO60, the relaxation of the published cross-section limits can be up to four orders of magnitude. Further we extended the developed method which now allows to do a combined analysis of several experiments. The combination of experiments can strengthen the limits by up to four orders of magnitude.

\footnotetext{
*** The European Physical Society Conference on High Energy Physics (EPS-HEP2021), ***

*** 26-30 July $2021 * * *$

*** Online conference, jointly organized by Universität Hamburg and the research center DESY ***
}

\footnotetext{
${ }^{*}$ Speaker
} 


\section{Introduction}

For dark matter (DM) particles with spin up to $1 / 2$, the non-relativistic effective field theory (NREFT) depends on 14 independent interaction operators, resulting in 28 coupling strengths for DM-nucleon interactions, since we assume contact interactions. Several direct detection (DD) experiments located in underground laboratories on the Earth were built to detect the so-called "Weakly Interacting Massive Particle", a promising dark matter candidate, by measuring the recoil energy of the target nucleon. So far any observed excess of such recoil events has been ruled out. However, limits on the DM-nucleon coupling strengths or cross-sections can be set. In order to determine the corresponding upper limits, collaborations commonly assume equal coupling of DM particles to protons and neutrons. Since this is not necessarily true, the provided limits cannot be straightforwardly applied to model predictions. In [1], we developed a method to determine upper limits on the DM-nucleon coupling strengths and cross-sections taking into account the interference of operators in the NREFT. In [2], we extended this method which now allows a combined analysis of several experiments. To apply the developed methods, we used data provided by the XENON1T [3] and PICO60 [4, 5] collaborations. The effect of operator interference can cause a relaxation of the published cross-section limits by up to four orders of magnitude. Due to the complementarity of target material, the combination of experiments can strengthen the published cross-section limits by up to four orders of magnitude.

\section{DM-nucleon scattering rate in the NREFT}

Following [6, 7], the generalized Hamiltonian for a set of DM-nucleon interactions writes as

$$
\mathcal{H}=\sum_{i} c_{i}^{p} \widehat{O}_{i}^{p}+c_{i}^{n} \widehat{O}_{i}^{n}
$$

Considering DM particles with spin up to $1 / 2$, there are 14 independent interaction operators $\widehat{O}_{i}^{p}\left(\widehat{O}_{i}^{n}\right)$ for the DM-proton (neutron) interaction of type $i$. The corresponding coupling strength is $c_{i}^{p}\left(c_{i}^{n}\right)$. Due to the linearity of the Hamiltonian in the coupling strengths, the interaction rate $R$ can be cast as

$$
R=\mathbf{c}^{T} \operatorname{Rc},
$$

where $\mathbf{c}$ is a 28 -dimensional vector containing the coupling strengths and $\mathbb{R}$ is a $28 \times 28$ matrix which depends on the detector material, DM velocity distribution and mass, and the local DM density. Using the upper limit on the interaction rate, $R^{u . l} \geq R$, which is provided by experimental collaborations, we can constrain the coupling strengths.

\section{Conservative approach}

Published results typically show the case of only one interaction at a time and $c_{i}^{p}=c_{i}^{n}$, but DM models generically lead to several interactions which interfere among each other. The common assumption might result in the exclusion of parameter space which is still allowed by data. Such a case is illustrated in Fig. 1. We specify the couplings $c_{\alpha}$ and $c_{\beta}$ to be $c_{i}^{0}$ and $c_{i}^{1}$, where " 0 " 


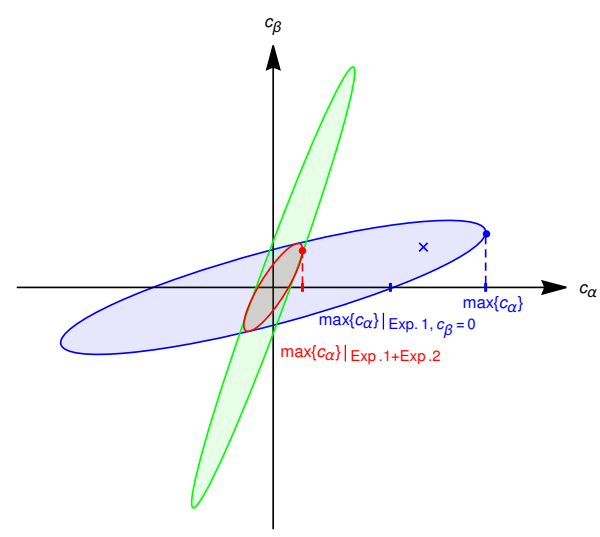

Figure 1: Parameter space spanned by the couplings $c_{\alpha}$ and $c_{\beta}$. The allowed parameter space for an experiment at a certain C.L. following the condition $R^{u . l .} \geq R$ is illustrated as ellipse. For experiment 1 (blue), the upper limit for $c_{\alpha}$ where $c_{\beta}=0$ is denoted as $\max \left\{c_{\alpha}\right\}_{\operatorname{Exp} .1, c_{\beta}=0}$. This limit excludes the blue cross, which is still allowed by data and not excluded by the conservative limit, which is denoted as $\max \left\{\mathrm{c}_{\alpha}\right\}$. The same logic applies for experiment 2 (green). The combined parameter space at a certain C.L. is displayed as red ellipse. The related upper limit is denoted as $\left.\max \left\{c_{\alpha}\right\}\right|_{\text {Exp.1+Exp.2. }}$

("1") means "isoscalar" ("isovector"). This so-called "isospin basis" is another way to describe the interactions among DM and the nucleus. The relations of the isospin and proton-neutron bases read $c_{i}^{p}=\left(c_{i}^{0}+c_{i}^{1}\right) / 2$ and $c_{i}^{n}=\left(c_{i}^{0}-c_{i}^{1}\right) / 2$. Assuming $c_{i}^{p}=c_{i}^{n}$, we get the upper limit $\left.\max \left\{c_{\alpha}\right\}\right|_{\operatorname{Exp} .1, c_{\beta}=0}$. However, there is parameter space which is still allowed according to data beyond this limit. This means that the blue cross would be mistakenly excluded by published limits. Our aim was to develop a method to get the most conservative limit, which is denoted as $\max \left\{c_{\alpha}\right\}$. It can be calculated by the compact expression [1]

$$
\max \left\{c_{\alpha}\right\}=\sqrt{\left(\mathbb{R}^{-1}\right)_{\alpha \alpha} R^{u . l}} .
$$

\section{Combined analysis}

Fig. 1 shows two ellipses with complementary eccentricities. As stated before, the detector material influences the properties of the $\mathbb{R}$-matrix and therefore the eccentricity of the ellipse. This illustrates that the parameter space allowed by the experiments at a certain C.L. (red) can be significantly constrained by the proper choice of experiments. In an ongoing project [2], we developed a method to determine the conservative upper limit of the combined parameter space at a certain C.L., which is denoted as $\left.\max \left\{c_{\alpha}\right\}\right|_{\text {Exp.1+Exp.2. The needed input are the R-matrices, and }}$ the number of observed events and background events of the considered experiments.

\section{Summary and conclusions}

In Fig. 2, we present results for the spin-dependent (SD) DM-proton cross-section, which is related to the coupling $c_{4}^{p}$, using data from XENON1T [3] (blue) and PICO60 [4, 5] (green). Furthermore, we assumed a Maxwell-Boltzmann velocity distribution for the DM particles and a 


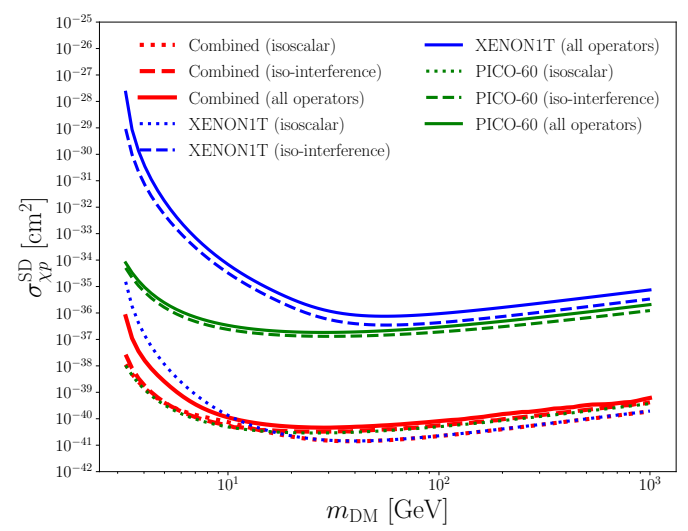

Figure 2: Upper limits on the SD DM-proton cross-section at $90 \%$ C.L. for different DM masses using data from XENON1T (blue) and PICO60 (green). We considered the scenarios "isoscalar" (dotted), "isointerference" (dashed) and "all operator" interference (solid). The limits for the combined analysis are displayed in red.

local DM density of $0.3 \mathrm{GeV} / \mathrm{cm}^{3}$. We considered the scenarios of "isoscalar" interactions (dotted), i.e. $c_{i}^{p}=c_{i}^{n}$, "iso-interference" (dashed), i.e. interference between $c_{i}^{p}$ and $c_{i}^{n}$ for a single operator $\widehat{O}_{i}$, and "all operators" (solid), i.e. interference between $c_{i}^{p}$ and $c_{i}^{n}$, and interference between all operators $\widehat{O}_{i}$. The effect of operator interference results in a relaxation of the published limits by roughly four orders of magnitude, whereas the combination of the experiments strengthens the limit by about four orders of magnitude. The results for other the other couplings are presented in [2].

\section{References}

[1] A. Brenner, A. Ibarra and A. Rappelt, Conservative constraints on the effective theory of dark matter-nucleon interactions from IceCube: the impact of operator interference, JCAP 2021 012, [arXiv:2011.02929v3]

[2] A. Brenner, G. Herrera, A. Ibarra, S. Kang, S. Scopel and G. Tomar, 2022 to be submitted to JCAP 2022

[3] E. Aprile et al., First Dark Matter Search Results from the XENONIT Experiment, 2017 Phys. Rev. Lett. 119 181301, [arXiv:1705.06655]

[4] C. Amole, Dark Matter Search Results from the PICO-60 CF $\mathrm{CF}_{3}$ Bubble Chamber, 2015 submitted to Phys. Rev. D, arXiv: 1510.07754

[5] C. Amole, Dark Matter Search Results from the Complete Exposure of the PICO-60 $\mathrm{C}_{3} \mathrm{~F}_{8}$ Bubble Chamber, 2019 Phys. Rev. D 100 022001, [arXiv: 1902.04031]

[6] N. Anand, A. Fitzpatrick and W. Haxton, Weakly interacting massive particle-nucleus elastic scattering response, Phys. Rev. C 89 no. 6 065501, [arXiv:1308.6288]

[7] A. Fitzpatrick, W. Haxton, E. Katz, N. Lubbers and Y. Xu, The Effective Field Theory of Dark Matter Direct Detection, 2013 JCAP 2013 004, [arXiv:1203.3542] 